\title{
Soziale Netzwerke und gesundheitliche Ungleichheiten im Alter
}

\author{
Britta Müller und Lea Ellwardt
}

\section{Überblick}

- Gesundheit und soziale Netzwerke sind im Alter die zentralen Lebensbereiche.

- Es existieren drei Thesen zum Verlauf des sozialen Gradienten von Gesundheit im Alter: die Kontinuitäts-, die Divergenz- und die Konvergenzthese.

- Eine der wichtigsten Thesen zum sozialen Netzwerk im Alter ist die Aktivitätsthese. Sie besagt, dass gute Lebenszufriedenheit durch das Beibehalten von sozialen Interaktionen erreicht werden könne.

- Sterblichkeits-, Demenz- und Depressionsrisiko sind sowohl mit sozioökonomischem Status als auch mit sozialen Netzwerkmerkmalen assoziiert.

- Die Vermittlungsmechanismen von sozioökonomischem Status, Gesundheit und sozialem Netzwerk im Alter können durch vorliegende Studien noch nicht hinreichend erklärt werden.

- Mehrheitlich werden Netzwerkmerkmale indirekt durch Proxys erhoben. Etablierte quantitative und qualitative Verfahren der Netzwerkanalyse spielen in der gerontologischen Forschung bislang eine untergeordnete Rolle.

B. Müller $(\bowtie)$

Rostock, Deutschland

E-Mail: britta.mueller@med.uni-rostock.de

L. Ellwardt

Köln, Deutschland

E-Mail: ellwardt@wiso.uni-koeln.de 
- Forschungsdesigns, die sich lediglich auf qualitative oder quantitative Netzwerkmerkmale konzentrieren, unterschätzen systematisch den eigentlichen Effekt der sozialen Einbettung von Personen im Alter auf die Gesundheit.

\section{$1 \quad$ Einleitung}

Durch Verbesserungen des Lebensstandards und des Gesundheitsverhaltens sowie durch den medizinischen Fortschritt seit der zweiten Hälfte des 20. Jahrhunderts ist das Alter zu einer eigenständigen Lebensphase geworden. Diese wird in der Regel durch den Übergang vom Erwerbsleben in den Ruhestand eingeleitet (Kohli 2000). Sowohl die Chance, den Ruhestand zu erreichen, als auch die fernere Lebenserwartung nach Eintritt in den Ruhestand haben sich deutlich erhöht (Eisenmenger und Emmerling 2011). Die nachberufliche Lebensphase umfasst bei vielen Menschen inzwischen mehrere Jahrzehnte. Zudem sind Personen, die in den Ruhestand wechseln, erheblich gesünder als Gleichaltrige früherer Geburtsjahrgänge (Crimmins 2004). Die Ausweitung dieser Lebensphase ging mit einer Differenzierung alter Menschen hinsichtlich Gesundheit und Selbstständigkeit einher: zu ihr gehören gesunde und aktive Menschen genauso wie hilfe- und pflegebedürftige Personen. Diesem Umstand wird dadurch Rechnung getragen, dass zwischen Personen im höheren und hohen Alter unterschieden wird (Baltes 2007). Merkmale des höheren Alters sind das Fehlen nichtkompensierbarer gesundheitlicher Einschränkungen, die Selbstgestaltung vielfältiger Aktivitäten (z. B. Reisen, Hobbies, Ehrenamt) sowie eine gute soziale Einbindung. Die Anforderungen des Alters können in dieser Phase insgesamt gut bewältigt werden. Das hohe Alter ist durch eine Zunahme körperlicher und kognitiver Einbußen sowie Krankheiten gekennzeichnet. Fähigkeiten und Möglichkeiten der Kompensation von Defiziten werden geringer (Baltes 1997; Baltes und Smith 2003).

Gesundheit und soziale Netzwerke werden im Alter zu zentralen Lebensbereichen und weisen im Vergleich $\mathrm{zu}$ früheren Lebensphasen Besonderheiten auf. Das Risiko für Erkrankungen mit langsamen Verläufen, die sich oft nicht vollständig heilen lassen, steigt. Als Ursachen dafür werden die Zunahme altersphysiologischer Prozesse, die Kumulation von Risikofaktoren im Lebensverlauf, lange Latenzzeiten und Folgeerkrankungen von bereits im frühen Lebensalter ausgebrochenen Erkrankungen betrachtet (Tesch-Römer und Wurm 2009). Zudem tritt Multimorbidität, also das gleichzeitige Auftreten mehrerer 
chronischer Erkrankungen, mit zunehmendem Alter häufiger auf, wodurch die funktionale Gesundheit gefährdet wird (Müller et al. 2014). Weiterhin nimmt der Stellenwert subjektiver Gesundheit im Alter zu. Im Vergleich zur objektiven Gesundheit ist sie im Alter ein zuverlässigeres Maß für Lebensqualität, Lebenserwartung sowie für die Prognose von Krankheitsverläufen. Im Vergleich zu Krankheitsdiagnosen spiegelt die subjektive Gesundheit, in die neben der physischen und psychischen Gesundheit auch die Lebenssituation und der Lebensstil einfließen, den individuellen Gesundheitszustand umfassender wider (Spuling et al. 2017).

Die strukturellen und funktionalen Muster sozialer Netzwerke im Alter werden v. a. durch den Statusübergang vom Erwerbsleben in den Ruhestand und durch die Gesundheit der älteren Person selbst als auch durch die ihrer Netzwerkpartner geprägt. Insbesondere in der Bedeutung der Gesundheit für das soziale Netzwerk unterscheidet sich diese Lebensphase von früheren, wie dem jungen oder mittleren Erwachsenenalter (Hoogendijk et al. 2016). Der Tod wichtiger Netzwerkpersonen, funktionale Einschränkungen oder auch Pflegebedürftigkeit verändern das soziale Netzwerk im Alter bedeutsam. So beträgt beispielsweise die Abnahme der Netzwerkgröße ab dem Alter von 60 bis 65 Jahren schätzungsweise eine Kontaktperson pro Dekade (Wrzus et al. 2013).

\section{Theorien}

\subsection{Theorien zur gesundheitlichen Ungleichheit im Alter}

Annahmen zum Zusammenhang zwischen sozialer Ungleichheit und Gesundheit im Alter werden in drei Thesen diskutiert. Die Kontinuitätsthese geht von einer Konstanz gesundheitlicher Ungleichheit aus: das Ausmaß gesundheitlicher Ungleichheit im Alter entspricht dem früherer Lebensphasen (Atchley 1989). Sie unterstellt, dass sich die soziale Ungleichheit im Ruhestand aus der sozialen Ungleichheit der Erwerbsphase reproduziere (Kohli et al. 2000). Die sozioökonomische Position, die eine Person im Laufe ihres Lebens über das Beschreiten von Bildungs- und Berufspfaden erreicht, würde im Alter beibehalten werden. Das impliziert eine zeitliche Stabilität sozialer Ungleichheit innerhalb einer Kohorte bis in das hohe Alter (Status-Maintenance-Hypothesis) (Henretta und Campbell 1976). Angenommen wird weiter ein konstanter Effekt sozialer Ungleichheit auf den Gesundheitszustand. Die Divergenzthese geht davon aus, dass sich die gesundheitliche Ungleichheit im Alter vergrößert. Dies sei Folge 
einer zunehmenden Kumulation gesundheitlicher Risiken bei unteren Statusgruppen (Tews 1993). Weiterhin wird die Divergenz darauf zurückgeführt, dass im Falle eines schlechten Gesundheitszustandes Ressourcen (wie z. B. das Einkommen), die kompensatorisch genutzt werden können, soziostrukturell unterschiedlich verfügbar seien (Mayer und Wagner 2010). Dieser Prozess werde bei Kumulation materieller Ressourcen höherer Statusgruppen während des Lebensverlaufs (Cumulative-Advantage-Hypothesis) noch verstärkt (Lampert et al. 2017). Die gegenteilige Position vertritt die Konvergenzthese. Sie besagt, dass sich die gesundheitliche Ungleichheit im Alter verringere. Zur Begründung dieser These werden vier Argumente angeführt. Erstens wird auf die Universalität biologischer Alterungsprozesse verwiesen, durch die der Einfluss sozialer Faktoren auf Gesundheit und Lebenserwartung zunehmend in den Hintergrund trete (Age-As-Leveler-Hypothesis) (Lampert et al. 2017; Mayer und Wagner 2010). Zweitens könnten wohlfahrtsstaatliche Regelungen Unterschiede im sozioökonomischen Status reduzieren bzw. dessen Einfluss auf die Gesundheit verringern (von dem Knesebeck et al. 2003). Drittens wird die These damit begründet, dass Belastungen des mittleren Lebensalters (z. B. als Folge der Berufstätigkeit), die schichtspezifisch variieren und den Gesundheitszustand beeinflussen, im Ruhestand an Bedeutung abnehmen (House et al. 1992). Viertens schließlich wird die Konvergenztheorie mit sozial selektiver Mortalität begründet: Das Risiko, vor Erreichen des Rentenalters zu versterben, ist in unteren Statusgruppen größer als in höheren. Damit stellen die Überlebenden der unteren Statusgruppen eine positive Selektion im Hinblick auf den Gesundheitszustand dar (Markides und Machalek 1984; McMunn et al. 2008).

\subsection{Theorien zu sozialen Netzwerken im Alter}

Eine frühe soziologische Theorie zu sozialen Netzwerken im Alter ist die vom Strukturfunktionalismus geprägte Disengagement-Theorie (Cumming und Henry 1961). Ihr liegt die Annahme zugrunde, dass eine gute Anpassung an das Alter über den Weg des „Disengagements“, verstanden als Rückzug älterer Menschen aus sozialen Rollen und Beziehungen, zustande käme. Unterstellt wird eine funktionale Komplementarität zwischen Individuum und Gesellschaft. Der Wunsch nach Rückzug entspräche dem Bedürfnis der Gesellschaft, ihr fortlaufendes Funktionieren durch rechtzeitige Rollenübernahme zu gewährleisten. Der Prozess des Disengagements sei weder auf einen schlechten Gesundheitszustand noch auf Einkommenseinbußen im Alter zurückzuführen. Vielmehr setze er bereits bei Aufgabe der Berufsrolle oder mit der Verwitwung ein. Lebenszufriedenheit werde dadurch 
erlebt, dass durch den Rückzug die soziale Kontrolle, etwa durch Kollegen und Vorgesetzte, reduziert werde, was eine Befreiung von Alltagsnormen mit sich bringe. Nur in jenen Fällen, in denen keine alternativen sozialen Rollen wie z. B. in Form des Ehrenamtes zur Verfügung ständen, würde die Reduktion der Zahl oder der Unterschiedlichkeit sozialer Kontakte als Krise erlebt werden. Die empirische Evidenz dieser Theorie ist gering. Zwar entfallen mit dem Ende der Berufstätigkeit und durch Witwenschaft in der Tat wichtige soziale Rollen, dafür werden jedoch bestehende soziale Kontakte, etwa zu Kindern, Enkelkindern und Nachbarn nicht etwa gelockert, sondern im Gegenteil, häufig intensiviert. Ein freiwillig initiierter Rückzug aus sozialen Bindungen bei guter Gesundheit im Alter ist nicht typisch (Maddox und Eisdorfer 1972; Neugarten et al. 1969; Shanas et al. 1968).

Als Gegenentwurf zur Disengagement-Theorie kann die Aktivitätstheorie betrachtet werden. Sie besagt, dass eine gute Lebenszufriedenheit im Alter nur durch fortgesetzte soziale Aktivität, das Beibehalten von Interaktionen bzw. eines aktiven Lebensstils erreicht werden könne. Dem altersbedingten Wegfall sozialer Rollen (z. B. Berufstätigkeit) und sozialer Aktivitäten sei mit der Aufnahme neuer Aktivitäten (z. B. Ehrenamt) zu begegnen (Tartler 1961). Dieser Zusammenhang zwischen Aktivität und Zufriedenheit im Alter ist empirisch gut gesichert (Adams et al. 2011; Katz 1996; Lemon et al. 1972).

Nach der zur Rational Choice Theory gehörigen Theorie des sozialen Austauschs kommen Interaktionen durch den über Normen geregelten Austausch von sozialen Gütern (instrumentelle, emotionale und materielle) zustande. Als zentral wird die Reziprozitätsnorm betrachtet. Mit Bezug auf das Alter geht die Theorie davon aus, dass ältere Menschen zunehmend weniger Ressourcen wie soziale Position, Geld und Gesundheit aufwiesen und dadurch für potenzielle Tauschpartner an Attraktivität verlören (Bengtson und Dowd 1981). Auswege aus dem drohenden Ungleichgewicht zwischen Nehmen und Geben werden in der Konzentration auf jene Interaktionspartner gesehen, mit denen Reziprozität möglich ist, sei es durch gezielte Selektion bestehender oder Aufnahme neuer Beziehungen, was letztlich als positiv für das Wohlbefinden erachtet wird. Die Kritik gegenüber dieser Theorie richtet sich v. a. auf die Schwierigkeit, die Annahmen empirisch zu überprüfen, da ,soziale Güter“ individuell sehr Unterschiedliches bedeuten können. Zudem hängt die Interpretation von Reziprozität auch von der Qualität und Bedeutung sozialer Beziehungen ab. Diese aber bleiben in der Theorie des sozialen Austauschs unberücksichtigt (Tesch-Römer 2010).

Spezifisch auf die Beziehungen zwischen Eltern im höheren bzw. hohen Alter und ihren erwachsenen Kindern sind das Modell der intergenerationalen Solidarität (Bengtson und Roberts 1991) und das Modell der intergenerationalen Ambivalenz. (Lüscher 2000) ausgerichtet. Ersteres fokussiert die wechselseitige 
Unterstützung zwischen den Generationen einer Familie und beschreibt diese anhand von sieben Dimensionen: „Struktur“ (im Sinne von Opportunitätsstrukturen für Interaktionen), „Assoziation“ (Ausmaß an persönlichen Kontakten), „Affekt“ (Ausmaß an gegenseitiger Zuneigung), „Konsens“ (Ausmaß der Übereinstimmung von Werten und Einstellungen), ,normative Überzeugungen“ (Stärke der Verbindlichkeit), „funktionaler Austausch“ (Grad gegenseitiger Unterstützung) und „Konflikt“ (Häufigkeit von Konflikten). Zweiteres geht von einer Widersprüchlichkeit sozialer Beziehungen zwischen Eltern und erwachsenen Kindern aus und formuliert Annahmen darüber, wie diese aufgelöst werden kann.

Aussagen zur strukturellen Veränderung sozialer Netzwerke im Alter treffen explizit die sozio-emotionale Selektivitätstheorie (Carstensen 1993) und das Modell des sozialen Konvois (Antonucci et al. 1997). Erstere Theorie mutmaßt, dass Lebenszufriedenheit und positive Gefühle trotz kleiner werdender Netzwerke bis ins hohe Alter deshalb stabil bleiben, weil zunehmend eine Konzentration auf jene Interaktionspartner erfolge, die Quelle positiver Erfahrungen seien. Dieser Selektionsprozess wird als funktional für die psychische Gesundheit angesehen. Die zweite Theorie nimmt an, dass der innere Kern des Netzwerkes hierzu gehören Partner, Kinder, Eltern und enge Freunde - einen Menschen über den Lebenslauf begleitet, der äußere Kern - bestehend aus Nachbarn, Dienstleistern, Arbeitskollegen - jedoch von Substitution geprägt ist. Hier verkleinert sich das Netzwerk nicht per se, sondern es verändert sich vielmehr hinsichtlich seiner Zusammensetzung. So kann der Kontakt zu (ehemaligen) Arbeitskollegen abnehmen und der Kontakt zu Nachbarn und Pflegekräften zunehmen. Die Substitution ist u. a. Ausdruck sich wandelnder Opportunitäten und Bedürfnisse im hohen Alter. Laut des Modells des sozialen Konvois können somit auch entfernte, weniger emotional-regulierende Kontakte funktional für die körperliche und psychische Gesundheit im Alter sein bzw. werden.

\section{Soziale Ungleichheit und Gesundheit}

Während für das mittlere Erwachsenenalter Zusammenhänge zwischen sozioökonomischem Status und Gesundheit seit langem vielfach belegt sind, gilt das Alter diesbezüglich als vergleichsweise junges Forschungsgebiet (von dem Knesebeck und Vonneilich 2009). Die folgende Darstellung konzentriert sich auf drei Folgen sozialer Ungleichheit, die hauptsächlich im Alter relevant sind. Dies sind Unterschiede im Sterblichkeitsrisiko, in der altersbedingt abnehmenden kognitiven Funktionsfähigkeit und dem damit verbundenen Demenzrisiko sowie im Depressionsrisiko. 


\subsection{Sterblichkeitsrisiko}

Gemäß empirischer Ergebnisse aus dem Deutschen Alterssurvey (DEAS) hat der sozioökonomische Status auch im Alter einen fortbestehenden Einfluss auf die Gesundheit (Schöllgen et al. 2010; Spuling et al. 2017). Dies hängt wiederum potenziell mit sozioökonomischer Ungleichheit in der Sterblichkeit zusammen. So war laut Registerdaten 2003 das Sterblichkeitsrisiko bei männlichen deutschen Rentnern ab 65 Jahren im untersten sozioökonomischen Fünftel (Quintil) dreimal so hoch wie im obersten bzw. betrug die verbleibende Lebenserwartung 12,5 Jahre versus 20 Jahre (Shkolnikov et al. 2007). Yao und Robert (2008) fanden in ihrer US-amerikanischen Langzeitstudie ähnliche Disparitäten in einer älteren Population mit 1631 Menschen von mindestens 60 Jahren: Schwarze Ältere hatten eine schlechtere subjektive Gesundheit und ein höheres Sterblichkeitsrisiko als weiße Ältere. Dieser Unterschied ließ sich teilweise durch einen niedrigeren sozioökonomischen Status der schwarzen Älteren sowohl auf individueller als auch auf Nachbarschaftsebene erklären. Lleras-Muney (2005) zeigte mit US-amerikanischen Zensusdaten von 1960, 1970 und 1980 ebenfalls einen kausalen Zusammenhang auf: Mit jedem zusätzlichen Jahr Ausbildung sank die Sterblichkeitswahrscheinlichkeit bei Erwachsenen um 3,6\% innerhalb der nächsten zehn Jahre. Dieser Ungleichheitstrend ist nicht auf Deutschland und die USA beschränkt. Im internationalen Vergleich 22 europäischer Länder haben Mackenbach et al. (2008) eindrucksvoll gezeigt, dass Personen mit einem niedrigen sozioökonomischen Status systematisch einem höheren Sterblichkeitsrisiko ausgesetzt waren als Personen mit einem höheren Status. Der altersbereinigte statusbasierte Unterschied im Sterblichkeitsrisiko war in den osteuropäischen und baltischen Staaten besonders hoch; am niedrigsten war er in den südeuropäischen Staaten Italien und Spanien. Die Analyse bezog sich auf Sterberegisterdaten von knapp dreieinhalb Millionen verstorbener Menschen mit einem Mindestalter von 30 Jahren.

\subsection{Kognitive Verläufe und Demenzrisiko}

Konsens besteht darin, dass der Verlauf kognitiver Fähigkeiten - diese umfassen die Dimensionen „Sprachfähigkeit“, „Gedächtnis“, „Allgemeinbildung“, „Problemlösen“ und „Orientierung“ - im Alter eine hohe interindividuelle Variabilität aufweist (Christensen et al. 1994). Zudem gestaltet er sich in Abhängigkeit vom jeweiligen Leistungsbereich unterschiedlich. So sind altersbedingte Differenzen bei den Dimensionen „Problemlösen“ und „Gedächtnis“ 
deutlich ausgeprägter als bei der „Allgemeinbildung“ (Finkel et al. 2007; Reischies und Lindenberger 2010).

Wenn die Abnahme pathologisch verläuft, also über eine normale altersbedingte Abnahme der kognitiven Leistungsfähigkeiten hinausgeht, und medizinisch diagnostiziert ist, spricht man von Demenz. Demenz ist eine psychiatrische Kondition, die bei degenerativen und nichtdegenerativen Erkrankungen des Gehirns auftritt. Die Krankheit, die am häufigsten mit Symptomen der Demenz einhergeht, ist die Alzheimer-Krankheit. Charakteristisch für Demenz sind starke Beeinträchtigungen der Gedächtnisleistung (besonders des Kurzzeitgedächtnisses), der Sprache, der Motorik und manchmal der Persönlichkeitsstruktur. Das Demenzrisiko steigt mit dem Alter exponentiell an. So beträgt die Prävalenz der Demenz vom Alzheimertyp bei der Gruppe der 60-Jährigen schätzungsweise ein Prozent und verdoppelt sich danach alle fünf Jahre (Ferri et al. 2005). Ab dem 85. Lebensjahr ist das Risiko bereits drastisch erhöht und misst etwa $25 \%$. Die kognitiven Verläufe und das Risiko, an Demenz zu erkranken, eine mögliche, aber nicht zwingende Folge eines ungünstigen Verlaufs, sind durch eine Vielzahl sozialer und sozioökonomischer Faktoren bedingt (Müller und Kropp 2011, 2012).

Der diesbezüglich wohl stärkste Faktor ist Intelligenz bzw. intellektuelle Kapazität, in Surveys häufig gemessen am Bildungsabschluss. Die Hypothese der kognitiven Reserve (Liberati et al. 2012; Scarmeas und Stern 2003) geht davon aus, dass Personen mit einem höheren Bildungsabschluss über ein größeres Repertoire an Bewältigungsstrategien verfügen, die die Abnahme an kognitiven Funktionsfähigkeiten im Alter hinauszögern und abmindern, als Personen mit niedrigerem Bildungsabschluss. Dies beinhaltet, dass eher alternative Regionen im Gehirn aktiviert werden können, die bei Bedarf die Funktionen weniger leistungsfähiger, von Demenz oder Alzheimer betroffener Regionen übernehmen. Nicht Bildung per se, sondern das damit assoziierte intellektuelle Anregungspotenzial des sozialen und beruflichen Umfeldes werden als Erklärung dafür herangezogen (Gow et al. 2012; Lee und Chi 2016; Then et al. 2013; Wang et al. 2012).

Die Assoziation zwischen Bildung und Demenzrisiko ist empirisch vielfach belegt: Laut einer Metaanalyse über 69 Studien haben ältere Menschen mit einem niedrigen Bildungsabschluss ein 1,61-mal so hohes Risiko, an Demenz zu erkranken, wie ältere Menschen mit einer hohen Bildung (Meng und D'Arcy 2012). Ein interessanter Befund ist der sogenannte Hürdeneffekt: Größere kognitive Reserven zögern die Diagnose einer Demenz zwar hinaus, setzt die Demenz jedoch erst einmal ein, verläuft sie schneller als bei geringeren Reserven. Dies kommt dadurch, dass die kompensatorischen Prozesse der kognitiven Reserve die stattfindende Abnahme kognitiver Funktionen lange maskieren. Bis die 
Abnahme klinisch diagnostiziert ist, haben sich die pathologischen Prozesse aber womöglich schon länger manifestiert (Bruandet et al. 2008).

Die kognitive Reserve hängt eng mit einem stimulierenden sozialen Umfeld zusammen. Mehrere theoretische Ansätze postulieren daher explizit einen Zusammenhang zwischen sozialer Einbettung und kognitiver Funktionsfähigkeit. Allen voran konstatiert die Use-it-or-lose-it-Hypothese (Hultsch et al. 1999), dass das Gehirn - ähnlich einem Muskel - regelmäßig trainiert werden muss, um voll funktionsfähig zu bleiben. Soziale Beziehungen helfen dabei, indem sie den Menschen zu sozialen und körperlichen Aktivitäten stimulieren [körperliche Aktivität ist ein weiterer Prädiktor kognitiver Funktion (Fratiglioni et al. 2004)] und komplexen intellektuellen Input liefern (Schooler 1984). Die StressPuffer-Hypothese (Fratiglioni et al. 2004) unterstellt eine positive Wirkung emotional unterstützender Beziehungen in Krisenzeiten. Stress gilt als Alzheimer-fördernder Faktor, da er mit strukturellen Veränderungen im Hippocampus assoziiert ist (Wilson et al. 2003). Dieser Hypothese zufolge profitieren Menschen insbesondere von sogenannten funktionalen Netzwerken mit reichhaltigen Unterstützungsleistungen ungeachtet der eigentlichen Beziehungsanzahl bzw. Netzwerkgröße. Die Haupteffekt-Hypothese (Cohen 2004) vermutet, dass stark integrierte Menschen über mehr Motivation (auch: Normen und sozialen Druck), Wissen und Ressourcen für einen gesunden Lebensstil verfügen. Bei dieser Hypothese besitzen auch sogenannte strukturelle Aspekte Relevanz, wie die Einbettung in komplexe und diverse Beziehungsgeflechte. Alle drei Hypothesen sind in ihren Aussagen komplementär, da sie sich auf unterschiedliche Mechanismen konzentrieren, die prinzipiell parallel wirksam sein können.

\subsection{Depression}

Depressive Störungen sind durch einen über einen längeren Zeitraum anhaltenden Zustand deutlich gedrückter Stimmung, Interesselosigkeit und Antriebsminderung gekennzeichnet. Im Alter sind Depressionen die häufigste psychische Störung überhaupt. Luppa und Kollegen (2012) berichten als Ergebnis ihrer Metaanalyse über älteren Menschen ab 75 Jahren Punktprävalenzen von $17 \%$ für eine depressive Symptomatik und von $7 \%$ für eine Major Depression. Depressionen im Alter erhöhen die suizidale Mortalität, gehen mit Einbußen der subjektiven und funktionalen Gesundheit einher und beeinträchtigen häufig das Behandlungsergebnis bei somatischen Erkrankungen. Sie sind Risikofaktor für die koronare Herzkrankheit (Carney und Freedland 2017). Es bestehen Zusammenhänge zwischen Depressionen und neurodegenerativen Erkrankungen wie Alzheimer-Demenz und 
Parkinson (Mourao et al. 2016, den Brok et al. 2015). Chronische Schmerzen im Alter erhöhen das Risiko einer Depression (Zis et al. 2017). Lorant et al. (2003) fanden in ihrer Metaanalyse, die auch Personen im Alter einschloss, überzeugende Belege dafür, dass ein geringer sozioökonomischer Status mit einem höheren Risiko für depressive Erkrankungen assoziiert ist. Obwohl in den eingeschlossenen Studien beide Richtungen des Zusammenhangs (der sozioökonomische Status beeinflusst das Depressionsrisiko im Sinne der Verursachungsthese; Depression bedingt den sozioökonomischen Status im Sinne der Driftthese) auftreten, sprechen die meisten Befunde dieser Metaanalyse für die Verursachungsthese. Aktuelle Studien zum Alter bestätigen den Befund eines sozialen Gradienten bei Depression (Domènech-Abella et al. 2018; Han et al. 2018; Lei et al. 2014; McEniry et al.2018). Besonders interessant ist das Ergebnis einer japanischen Studie, in der die Autoren die späte Wirkung früher Lebenserfahrungen nachweisen konnten: Personen, die in Familien mit einem geringen sozioökonomischen Status aufgewachsen waren, hatten Jahrzehnte später - im Alter - ein um 44 \% höheres Risiko, an einer Depression zu erkranken als jene, deren Eltern einen hohen sozioökonomischen Status aufgewiesen hatten (Tani et al. 2016).

\section{$4 \quad$ Soziale Netzwerke und Gesundheit}

In der Forschung werden eine Vielzahl von Gesundheitsparametern in ihrem Bezug zu sozialen Netzwerken im Alter untersucht, wobei - den Besonderheiten des Alters entsprechend - der Fokus besonders häufig auf dem Sterblichkeitsrisiko, kognitiven Verläufen und Depression liegt. Zu diesen drei Schwerpunkten werden im Folgenden ausgewählte Studien dargestellt.

\subsection{Sterblichkeitsrisiko}

Die Einbettung in soziale Netzwerke ist mit dem Sterblichkeitsrisiko assoziiert. $\mathrm{Zu}$ dieser Schlussfolgerung kam die Forschergruppe um Holt-Lunstad in ihrer Metaanalyse von 70 Studien zu subjektiver und objektiver sozialer Isolation (Holt-Lunstad et al. 2015): Einsamkeit sowie Alleinleben korrespondierten in ähnlicher Assoziationsstärke mit einem erhöhten Sterblichkeitsrisiko (26\% und $32 \%$ ). Dieses Ergebnis knüpft an eine frühere Metaanalyse derselben Erstautorin an: Über 148 Studien hinweg fand sie eine um $50 \%$ höhere Sterbewahrscheinlichkeit für schwach im Vergleich zu stark eingebetteten Personen (Holt-Lunstad et al. 2010). Einbettung wurde über funktionale (bspw. Erhalten sozialer Unterstützung, Einsamkeit) und strukturelle Netzwerkmaße (bspw. Anzahl sozialer 
Beziehungen, Haushaltsgröße) gemessen. Dieser Unterschied in der Sterbewahrscheinlichkeit, welcher in etwa mit dem Gesundheitsrisiko des Rauchens und der Adipositas dritten Grades vergleichbar ist, zeigte sich konsistent über Altersgruppen, Geschlecht, ursprünglichen Gesundheitszustand, Todesursache und Beobachtungszeitraum der Studien hinweg. Interessante Differenzen stellten sich dabei bezüglich der verwendeten Netzwerkmaße heraus: Der Zusammenhang zwischen Einbettung und Sterblichkeit war am größten, wurden funktionale und strukturelle Maße von Netzwerken kombiniert.

\subsection{Kognitive Verläufe und Demenzrisiko}

Einige Metaanalysen haben bereits die beeindruckende Vielzahl an Studien zu sozialer Integration und kognitiver Funktion zusammenfassend aufgearbeitet. In einer Metaanalyse von Kuiper et al. (2015) zeigten drei von 43 Studien einen signifikanten Zusammenhang zwischen kognitiver Funktionsabnahme und Netzwerkgröße. Ältere Menschen mit kleineren Netzwerken verzeichneten eine stärkere Abnahme im Beobachtungszeitraum als ältere Menschen mit größeren Netzwerken (Chi und Chou 2000; Holtzman et al. 2004; Hughes et al. 2008). Diese Assoziation war stärker als für funktionale Aspekte wie geringe soziale Aktivität.

In einer weiteren Übersicht zu 19 Längsschnittstudien fanden Kuiper und Kollegen (2015) einen positiven Zusammenhang zwischen Demenzrisiko und geringer sozialer Teilhabe, geringer Kontakthäufigkeit und hoher Einsamkeit. Die Autoren verglichen die Effektstärken mit denen einer geringen Bildung, geringer körperlicher Aktivität und der Altersdepression. Ergebnisse zu Netzwerkgröße und Zufriedenheit mit dem Netzwerk blieben hingegen inkonsistent, sodass hierfür kein signifikanter Zusammenhang gefunden wurde: Nur zwei der acht Studien, die Netzwerkgröße berücksichtigten, zeigten eine erhöhte Demenzwahrscheinlichkeit von älteren Menschen mit kleinen Netzwerken (James et al. 2011; Saczynski et al. 2006).

$\mathrm{Zu}$ einem ähnlichen Ergebnis kommen Fratiglioni und Kollegen (2004) in ihrer Metaanalyse von 13 Studien. Drei von sechs Studien, die soziale Netzwerke analysierten, fanden ein vermindertes Demenzrisiko für stark sozial integrierte Personen (Fratiglioni et al. 2000; Scarmeas et al. 2001; Wang et al. 2002). Fünf von sieben Studien fanden eine geringere Abnahme kognitiver Funktionsfähigkeiten. Darüber hinaus ist auch der umgekehrte Kausalzusammenhang belegt: Nehmen kognitive Fähigkeiten mit dem Alter stark ab, verkleinert sich infolgedessen oft auch das soziale Netzwerk der Betroffenen (Aartsen et al. 2004), da sich ihr sozialer und physischer Aktionsradius verringert. Die zunehmenden kognitiven Beeinträchtigungen können so einen Rückzug in familiäre Beziehungsnetzwerke begünstigen. 


\subsection{Depression}

In ihrem systematischen Review analysierten Schwarzbach et al. (2014) insgesamt 37 Studien, die Zusammenhänge zwischen sozialen Netzwerken und Depression bei älteren Menschen untersucht hatten. Während die Befunde zu funktionalen Netzwerkaspekten insgesamt sehr konsistent sind (wenig soziale Unterstützung und geringe Beziehungsqualität sind mit Depression assoziiert), fielen die zu strukturellen Netzwerkaspekten (wie Familienstand, Netzwerkgröße und Kontakthäufigkeit) mehrheitlich heterogen aus. Übereinstimmend zeigte sich hingegen, dass Alleinlebende kein höheres Risiko für depressive Symptome aufwiesen. Teilweise werden die Zusammenhänge durch den kulturellen Kontext moderiert. So war in östlichen Kulturen wie China und Japan eine hohe Kontaktfrequenz mit einem geringeren Depressionsrisiko assoziiert. Für westliche Kulturen hingegen konnte dieser Zusammenhang nicht bestätigt werden. Antonucci et al. (1997) fanden in ihrer Analyse Belege dafür, dass funktionale und strukturelle Netzwerkaspekte je eigenständige Wirkungen auf depressive Symptome im Alter haben. Der Bedeutung sozialer Netzwerke für die Beziehung zwischen funktionalen Beeinträchtigungen und depressiven Symptomen gingen Litwin und Stoeckel (2016) in ihrer Arbeit nach. Es zeigte sich, dass funktional beeinträchtigte Personen mehr depressive Symptome aufweisen, wenn sie über keinerlei soziales Netzwerk verfügten im Vergleich zu jenen mit Netzwerkpartnern.

\section{$5 \quad$ Soziale Ungleichheit, soziale Netzwerke und Gesundheit}

Studien, die Beziehungen zwischen sozialer Ungleichheit, sozialen Netzwerken und Gesundheit im Alter analysieren, sind vergleichsweise rar. Sie berücksichtigten sowohl funktionale (also v. a. Unterstützungsformen) als auch strukturelle (also v. a. Größe, Häufigkeit der Kontakte) Aspekte. Die Darstellung vorliegender Befunde strukturiert sich entlang dreier Hypothesen:

1. Merkmale sozialer Netzwerke mediieren den Zusammenhang von sozioökonomischem Status und Gesundheit, d. h. der sozioökonomische Status wirkt sich über soziale Netzwerke auf den Gesundheitszustand aus.

2. Merkmale sozialer Netzwerke moderieren den Zusammenhang von sozioökonomischem Status und Gesundheit, d. h. Netzwerkmerkmale beeinflussen die Stärke des Zusammenhangs zwischen sozioökonomischem Status und Gesundheitszustand. 
3. Der sozioökonomische Status moderiert den Zusammenhang von sozialem Netzwerk und Gesundheit, d. h. der sozioökonomische Status beeinflusst die Stärke des Zusammenhangs zwischen Netzwerkmerkmalen und Gesundheitszustand.

\subsection{Soziales Netzwerk als Mediator}

An einer deutschen Stichprobe von im eigenen Haushalt lebenden Personen im Alter $\geq 60$ Jahretestete von dem Knesebeck (2005) die Hypothese, dass soziale Netzwerke den Zusammenhang von sozioökonomischem Status und Gesundheit mediieren. Abhängige Variablen waren die subjektive Gesundheit, depressive Symptome und funktionale Einschränkungen. Der sozioökonomische Status wurde mittels Bildung, Einkommen und Beruf erfasst. Zur Abbildung des sozialen Netzwerkes wurden die beiden Proxys „Kontakthäufigkeit mit Freunden/Bekannten“ und „Kontakthäufigkeit mit Familienmitgliedern“ sowie die wahrgenommene Verfügbarkeit, Inanspruchnahme und Adäquatheit emotionaler Unterstützung verwandt. Im Ergebnis zeigte sich, dass die vermittelnden Effekte sozialer Netzwerke auf den sozialen Gradienten von Gesundheit insgesamt nur schwach sind: Bei Kontrolle der Items „Kontakthäufigkeit mit Freunden/Bekannten“, „Kontakthäufigkeit mit Familienmitgliedern“ und „Verfügbarkeit emotionaler Unterstützung" verringern sich die positiven Zusammenhänge zwischen Einkommenshöhe und subjektiver sowie funktionaler Gesundheit bzw. der negative Zusammenhang zwischen Einkommenshöhe und Depressivität nur leicht und bleiben im signifikanten Bereich. Eine Verstärkung zeigt sich gar bei den Zusammenhängen zwischen Bildung bzw. Beruf und den drei Gesundheitsindikatoren, wenn die Netzwerkmerkmale kontrolliert werden (von dem Knesebeck 2005). Depressive Symptome wurden auch in der koreanischen Studie von Han et al. (2018) untersucht. Sie konnten zeigen, dass der Grad der Reziprozität des sozialen Austauschs den Zusammenhang von Haushaltseinkommen und Depression mediiert. Die subjektive Gesundheit untersuchten Vonneilich et al. (2012) im Rahmen der deutschen Heinz Nixdorf Recall Studie. Die analysierten Daten stammen aus der Baseline-Untersuchung und dem Fünf-Jahres-Follow-up (4146 Männer und Frauen im Alter 45 bis 75 Jahre). Strukturelle Merkmale wurden mit dem „Social Integration Index“ (SNI) erhoben (Berkman et al. 2004). Funktionale Merkmale wurden mit dem „New Haven Established Population for Epidemiologic Studies of the Elderly Questionnaire“ (EPESE) gemessen (Seeman und Berkman 1988). Es konnte gezeigt werden, dass sowohl strukturelle als auch funktionale Netzwerkmerkmale Zusammenhänge zwischen 
dem sozioökonomischen Status und der subjektiven Gesundheit vermitteln. Im Gegensatz dazu traten in der prospektiven Follow-up-Studie (Beobachtungszeitraum drei Jahre) von Nilsson et al. (2010) keine Mediatoreffekte des sozialen Netzwerkes auf den Zusammenhang von sozioökonomischem Status (gemessen über das finanzielle Vermögen) und der funktionalen Gesundheit (gemessen über die Anzahl von Mobilitätsaktivitäten, die ohne fremde Hilfe ausgeführt werden können) auf. Eingesetzte Netzwerkindikatoren bezogen sich auf Kohabitation, soziale Teilhabe, Netzwerkdiversität und Zufriedenheit mit sozialen Kontakten (Nilsson et al. 2010). Auch die Ergebnisse von Yan et al. (2013) auf der Grundlage einer Follow-up Studie, die einen Beobachtungszeitraum von 11,5 Jahren umfasste, stützen die Mediatorhypothese nicht. Die amerikanische Stichprobe bestand aus 64- bis 100-jährigen Personen. Untersucht wurde die Häufigkeit eines ischämischen Schlaganfalls in Abhängigkeit vom sozioökonomischen Status des Wohnviertels (,Neighbourhood socioeconomic status“). Netzwerkmerkmale wurden mit der „Interpersonal Support Evaluation List“ (Cohen et al. 1985), die die wahrgenommene Verfügbarkeit von sozialer Unterstützung erfasst, und der „Lubben Social Network Scale“ (Lubben 1988), ein speziell für die ältere Bevölkerung entwickeltes Instrument, das neben der emotionalen und instrumentellen Unterstützung auch die tatsächliche Größe des Netzwerkes erfragt, erhoben.

In der Zusammenschau geben die referierten Befunde keine eindeutige Antwort auf die Frage nach einem Mediatoreffekt struktureller Netzwerkmerkmale im höheren Lebensalter.

\subsection{Soziales Netzwerk als Moderator}

Mit Daten der vierten Welle der SHARE-Studie (Survey of Health, Ageing and Retirement in Europe) untersuchten Olofsson et al. (2018) Moderatoreffekte sozialer Netzwerke. Die Stichprobe umfasste 54.751 Personen im Alter $\geq$ 50 Jahre aus 16 europäischen Ländern. Zentrale Indikatoren waren Bildung und subjektive Gesundheit. Der Erhebung von Netzwerkgröße und -zufriedenheit lag ein Netzwerkgenerator zugrunde. Als Ergebnis wird ein moderierender Effekt der Netzwerkzufriedenheit bei Männern und Frauen Nordeuropas berichtet: Bei hoher Zufriedenheit ist der Zusammenhang zwischen sozioökonomischem Status und Gesundheit stärker, bei geringer Zufriedenheit schwächer ausgeprägt. Die Autoren argumentieren, dass insbesondere Personen mit geringerer Bildung bei schlechter Gesundheit Hilfe im sozialen Netzwerk finden, was mit hoher Zufriedenheit einhergeht. Darüber hinaus wird vermutet, dass eine geringe Zufriedenheit mit Netzwerkkontakten Folge emotional belastender Beziehungen 
sein könne, die das Wohlbefinden schwäche und den sozialen Gradienten verringere (Olofsson et al. 2018).

Das Ergebnis dieser Studie stützt die Hypothese vom Moderatoreffekt sozialer Netzwerke auf den sozialen Gradienten von Gesundheit. Der soziale Gradient ist bei hoher Zufriedenheit mit dem sozialen Netzwerk stärker ausgeprägt.

\subsection{Sozioökonomischer Status als Moderator}

Mittels längsschnittlicher Daten der MacArthurStudy of Successful Aging untersuchten Unger et al. (1999) die Rolle des sozioökonomischen Status (erhoben über Einkommen) bezüglich des Einflusses sozialer Netzwerke auf Veränderungen funktionaler Gesundheit im Zeitraum von sieben Jahren. Die Stichprobe umfasste Personen im Alter von 70-79 Jahren. Die Netzwerk-Items bezogen sich auf strukturelle und funktionale Merkmale. Es zeigte sich, dass bei Personen mit einem größeren sozialen Netzwerk funktionale Beeinträchtigungen geringer ausfielen als bei Personen mit kleinem Netzwerk. Dieser Zusammenhang war bei Männern besonders ausgeprägt. Das Einkommen indes erwies sich nicht als Moderatorvariable. In der bereits erwähnten Studie von von dem Knesebeck (2005) wurden neben Mediatoreffekten auch Moderatoreffekte getestet. Diese fielen in Abhängigkeit von Gesundheitsindikator und Netzwerkmerkmal inkonsistent aus. Während die positiven Korrelationen zwischen Netzwerkmerkmalen (Kontakthäufigkeit mit Freunden; Kontakthäufigkeit mit Familie) und subjektiver Gesundheit bzw. funktionaler Gesundheit bei mittleren Statusgruppe schwächer als bei niedrigen und hohen Statusgruppen waren, erwies sich die negative Korrelation zwischen Kontakthäufigkeit und Depressivität bei Personen mit hohem Status am stärksten: Personen mit seltenen Kontakten zu Freunden und Familie und defizitärer sozialer Unterstützung hatten, wenn sie der hohen Statusgruppe angehörten, ein höheres Risiko, an Depression zu erkranken als Personen mit geringerem Status. Auch in der Heinz-Nixdorf Recall Studie (Vonneilich et al. 2011) zeigten sich nur wenige statistisch signifikante Interaktionseffekte von sozioökonomischem Status und sozialem Netzwerk auf die subjektive Gesundheit bzw. Depression, die zudem zwischen Männern und Frauen variierten.

Die berichteten Befunde unterstützen nur partiell die Annahme, dass der sozioökonomische Status die empirisch vielfach belegten Zusammenhänge zwischen strukturellen Netzwerkmerkmalen und Gesundheit im höheren Lebensalter moderiert. 


\section{Zusammenfassung und kritische Reflexion}

Der Beitrag konnte zeigen, dass auch im höheren Lebensalter Zusammenhänge zwischen sozioökonomischem Status und Sterblichkeits-, Demenz- und Depressionsrisiko bestehen. Die dargestellten Befunde geben jedoch keine eindeutige Antwort auf die Frage nach dem Verlauf gesundheitlicher Ungleichheit im Lebenslauf. Die referierten Studien bestätigen sowohl die Kontinuitäts- als auch die Divergenzthese. So deuten einerseits die Studien zum Sterblichkeitsrisiko darauf hin, dass sich im Lebensverlauf statusbedingte Unterschiede im Sterberisiko fortsetzen (Kontinuitätsthese). Das höhere Risiko von Personen mit geringerem sozioökonomischen Status, im Alter an Demenz und Depression zu erkranken, kann andererseits die gesundheitliche Ungleichheit vergrößern (Divergenzthese). Die Uneindeutigkeit der Befunde, bezogen auf den Verlauf gesundheitlicher Ungleichheit, wurde in der Forschungsliteratur häufig konstatiert. Dies ist auch den unterschiedlichen Operationalisierungen der sozioökonomischen Indikatoren (Bildung, Berufsstatus, Einkommen) und den verschiedenen Gesundheitsdimensionen geschuldet. Fraglich ist darüber hinaus, ob die herkömmlichen Indikatoren überhaupt geeignet sind, Unterschiede in den akkumulierten sozioökonomischen Bedingungen im Alter hinreichend $\mathrm{zu}$ erfassen (Ausbildung und Beruf liegen lange zurück, sind für Männer und Frauen nur bedingt vergleichbar, Eigentumsverhältnisse geben mehr Aufschluss über Anhäufung von Ressourcen) (Clemens 2008; von dem Knesebeck und Schäfer 2009; Kohli et al. 2000; Leopold und Engelhardt 2011). Für Eltern im hohen Alter ist z. B. denkbar, dass die gesundheitliche Ungleichheit auch auf ungleiche, sozioökonomisch bedingte Ressourcen ihrer erwachsenen Kinder (wie z. B. Ausmaß der Unterstützung bei Pflegeleistungen; Wissen über Diagnose- und Behandlungsmöglichkeiten) beeinflusst wird (Rueda und Artazcoz 2009; Saraceno 2010). Wie zudem am Beispiel der Depression gezeigt werden konnte, kann darüber hinaus auch der erlebte elterliche sozioökonomische Status in der Kindheit für die Gesundheit im Alter eine nicht zu unterschätzende Rolle spielen (Brandt et al. 2012; Pakpahan et al. 2017). Für weitere Forschungen kann es lohnend sein, neben der sozioökonomischen Position der Person im Alter auch die ihrer Eltern und erwachsenen Kinder stärker in den Blick zu nehmen.

Studienbefunde zu den Wechselwirkungen zwischen strukturellen Netzwerkmerkmalen und Sterblichkeits-, Demenz- und Depressionsrisiko stützen die Aktivitätstheorie: Die Pflege von sozialen Interaktionen auch im Ruhestand erweist sich als wichtiger Schutzfaktor für die Gesundheit. Es scheint, dass es dabei weniger auf die Netzwerkgröße als vielmehr auf das Ausmaß sozialer Einbettung ankommt. Diese kann, entsprechend der sozioemotionalen 
Selektivitätstheorie, auch bei abnehmender Netzwerkgröße erlebt werden. Eine Erklärung dafür könnte in der Stabilität der Netzwerkpersonen, die, gemäß der Theorie des sozialen Konvois, zum inneren Kern zählen, liegen. Für eine abschließende Diskussion ist jedoch mehr Forschung hinsichtlich Anzahl und Rollendiversität sozialer Beziehungen (Ellwardt et al. 2015), aber auch anderer struktureller Netzwerkcharakteristika wie Netzwerkdichte und Brückenbeziehungen erforderlich. Deutlich wurde zudem, dass bei der Analyse der Zusammenhänge zwischen sozialem Netzwerk und Gesundheit der kulturelle Kontext berücksichtigt werden muss (Li und Zhang 2015; Schwarzbach et al. 2014). Wichtig ist, dass sowohl funktionale als auch strukturelle Merkmale je eigenständige Beiträge zur Varianzaufklärung gesundheitlicher Parameter im Alter leisten können (Antonucci et al. 1999). Darüber hinaus wurde sichtbar, dass die Zusammenhänge mit Gesundheit v. a. dann groß waren, wenn komplexe Maße analysiert worden sind (z. B. sowohl Lebensform als auch Netzwerkintegration und Erleben der Netzwerkbeziehung). Dies bedeutet, dass Forschungsdesigns, die sich lediglich auf qualitative oder quantitative Netzwerkmerkmale konzentrieren, den eigentlichen Effekt der sozialen Einbettung potenziell systematisch unterschätzen können. Es sollten also mehrere Indikatoren parallel getestet (Ellwardt et al. 2015) oder aber kombiniert werden, z. B. in Form von Netzwerktypologien (Ellwardt et al. 2016). Bei der Bildung einer Typologie werden Personen anhand verschiedener Merkmale ihrer Netzwerke klassifiziert, z. B. in Gruppen mit großen funktionalen versus kleinen wenig funktionalen Netzwerken. Diese Personengruppen können in einem weiteren Schritt hinsichtlich ihrer Gesundheit verglichen werden. Kritisch anzumerken ist ferner, dass häufig lediglich Proxys zur Operationalisierung von sozialen Netzwerken herangezogen werden, wie z. B. die Häufigkeit sozialer Kontakte oder die Haushaltszusammensetzung. Dabei liegen durchaus Hinweise zum Mehrwert echter Netzwerkanalysen für die Erklärung von Gesundheit im Alter vor (Li und Zhang 2015; Schwarzbach et al. 2014; Youm et al. 2014). Längsschnittliche Analysen fokussieren bislang vor allem die Testung von Netzwerkeffekten auf Gesundheit. Forschungslücken bestehen bezüglich der Frage nach einer gegenteiligen Wirkrichtung, dem Einfluss gesundheitlicher Verschlechterungen auf soziale Netzwerke.

Vorliegende Analysen zu Zusammenhängen der drei Bereiche ,sozioökonomischer Status“, „Gesundheit“ und ,,soziales Netzwerk“ konzentrieren sich v. a. auf Depression sowie funktionale und subjektive Gesundheit. Das Sterblichkeitsrisiko und Demenzerkrankungen sind hingegen weitgehend unerforscht. Die Vermittlungsmechanismen von sozioökonomischem Status, Gesundheit und sozialem Netzwerk im Alter können durch bisherige Studien nicht hinreichend erklärt werden. Nach bisherigem Kenntnisstand erscheinen Moderatoreffekte von 
Netzwerkmerkmalen auf die gesundheitliche Ungleichheit im Alter am wahrscheinlichsten.

Aus der bisherigen Forschung wird folgendes deutlich: Erstens liegt der Fokus der Studien auf in der eigenen Häuslichkeit lebenden Personen. Weitgehend unklar ist, inwieweit vorliegende Befunde auch auf in Pflegeeinrichtungen lebende Personen übertragbar sind. Diese Forschungslücke gilt es zu schließen. Zweitens hat sich gezeigt, dass Designs, die komplexe Merkmale von sozialen Netzwerken erheben, für die Untersuchung der Beziehung ,,sozioökonomischer Status“, „Gesundheit" und „soziales Netzwerk" besser geeignet sind als Instrumente, die nur quantitative oder nur qualitative Merkmale erheben. Drittens werden Netzwerkmerkmale in der gerontologischen Forschung mehrheitlich nur indirekt, durch Proxys, gemessen. Etablierte quantitative und qualitative Verfahren der Netzwerkanalyse spielen bei der Erforschung von alten Menschen bislang eine untergeordnete Rolle. Ihr Potenzial sollte für zukünftige Forschung wesentlich stärker genutzt werden.

\section{Leseempfehlungen}

Vonneilich, N., Jöckel, K.-H., Erbel, R., Klein, J., Dragano, N., Siegrist, J., \& von Dem Knesebeck, O. (2012). The mediating effect of social relationships on the association between socioeconomic status and subjective health-results from the Heinz Nixdorf Recall cohort study. BMC Public Health. https://doi.org/10.1186/1471-2458-12-285 Die Studie ist eine der ersten, die den Effekt des SES auf die Beziehung zwischen sozialen Beziehungen und Gesundheit untersucht (Durchschnittsalter der Stichprobe: 60 Jahre). Es zeigen sich nur wenige statistisch signifikante Interaktionseffekte von sozioökonomischem Status und sozialem Netzwerk auf die subjektive Gesundheit bzw. Depression, die zudem zwischen Männern und Frauen variieren.

Ellwardt, L., van Tilburg, T., Aartsen, M., Wittek, R., \& Steverink, N. (2015). Personal networks and mortality risk in older adults: a twenty-year longitudinal study. PloS one. https://doi.org/10.1371/journal.pone.0116731 Mittels Daten aus der Longitudinal Aging Study Amsterdam (LASA) werden Beziehungen zwischen der Sterblichkeit und Netzwerkmerkmalen, die sowohl strukturelle als auch funktionale Aspekte abbilden, berichtet.

Goldman, A. W., \& Cornwell, B. (2015). Social network bridging potential and the use of complementary and alternative medicine in later life.Social Science \& Medicine. https://doi.org/10.1016/j.socscimed.2015.07.003 Die Studie testet mit Daten der 1. Welle des National 
Social Life, Health, and Aging Project (NSHAP) die Hypothese, dass Personen, die ansonsten unverbundene Gruppen miteinander verbinden, also eine Brückenfunktion innehaben, häufiger als andere Netzwerkmitglieder komplementäre Medizin in Anspruch nehmen.

\section{Datensätze}

- „SHARE“ (The Survey of Health, Ageing and Retirement in

\section{Europe)}

Die Studie begann 2004 als repräsentative Befragung der Bevölkerung im Alter 50+. An der Baseline-Untersuchung nahmen 11 europäische Länder (Belgien, Dänemark, Deutschland, Frankreich, Griechenland; Italien, Niederlande, Österreich, Schweiz, Schweden, Spanien) teil. Es liegen quer- und längsschnittliche Daten aus sechs Erhebungswellen und Teilnehmern aus 27 europäischen Ländern sowie Israel vor. In der vierten und sechsten Welle wurde das soziale Netzwerk über Namensgeneratoren erfragt.

www.share-project.org

\section{- „Heinz Nixdorf Recall Studie““}

Es handelt sich um eine bevölkerungsbasierte Kohortenstudie. Die Stichprobenteilnehmer leben in der Metropole Ruhr und waren zur Baseline-Untersuchung 2000-2003 45-75 Jahre alt. Eine zweite Welle fand 2006-2008, eine dritte 2010-2013 statt. Untersucht werden insbesondere kardiovaskuläre Erkrankungen. Eingesetzte Netzwerkinstrumente sind der "Social Integration Index“ (SII) und die deutsche Adaption des „New Haven Established Population for Epidemiologic Studies of the Elderly (EPESE) Questionnaire“. https://www.uni-due.de/recall-studie

\section{- „LASA“ (Longitudinal Aging Study Amsterdam)}

Die Studie fokussiert physische, emotionale und soziale Alternsprozesse anhand einer niederländischen Stichprobe. Zur Baseline-Untersuchung 1992 waren die Teilnehmer der Studie im Alter von 55-85 Jahren. Follow-Up-Untersuchungen erfolgen alle drei Jahre; in jeder Welle werden Daten zum persönlichen Netzwerk erhoben.

https://www.lasa-vu.nl/ 


\section{- „HRS“ (The Health and Retirement Study)}

Die Ausgangsstichprobe dieser amerikanischen Längsschnittstudie bildeten 1931 bis 1941 Geborene. Sie wurden erstmals 1992 untersucht. Erneute Untersuchungen finden alle zwei Jahre statt. Indikatoren zum sozialen Netzwerk erfassen dessen Zusammensetzung, die Anzahl enger Beziehungen sowie die Kontakthäufigkeit. https://hrs.isr.umich.edu/about

- „NSHAP“ (National Social Life, Health, and Aging Project)

Die Baseline-Untersuchung dieser amerikanischen Studie fand 20052006 statt; die Stichprobenteilnehmer waren im Alter von 57 bis 85 Jahren. 2010-2011 wurde eine zweite Welle realisiert. Über Namensgeneratoren werden egozentrierte Netzwerke erhoben.

http://www.norc.org/Research/Projects/Pages/national-social-life-health-and-aging-project.aspx

\section{Literatur}

Aartsen, M. J., Van Tilburg, T., Smits, C. H., \& Knipscheer, K. C. (2004). A longitudinal study of the impact of physical and cognitive decline on the personal network in old age. Journal of Social and Personal Relationships, 21(2), 249-266.

Adams, K. B., Leibbrandt, S., \& Moon, H. (2011). A critical review of the literature on social and leisure activity and wellbeing in later life. Ageing \& Society, 31(4), 683-712.

Antonucci, T. C., Fuhrer, R., \& Dartigues, J.-F. (1997). Social relations and depressive symptomatology in a sample of community-dwelling French older adults. Psychology and Aging, 12(1), 189.

Antonucci, T. C., Ajrouch, K. J., \& Janevic, M. (1999). Socioeconomic status, social support, age, and health. Annals of the New York Academy of Sciences, 896(1), 390-392.

Atchley, R. C. (1989). A continuity theory of normal aging. The Gerontologist, 29(2), 183-190.

Baltes, P. B. (1997). Die unvollendete Architektur der menschlichen Ontogenese: Implikation für die Zukunft des vierten Lebensalters. Psychologische Rundschau, 48, 191-210.

Baltes, P. B. (2007). Alter (n) als Balanceakt: Im Schnittpunkt von Fortschritt und Würde. In P. Gruss (Hrsg.), Die Zukunft des Alterns: Die Antwort der Wissenschaft (S. 15-34). München: Beck.

Baltes, P. B., \& Smith, J. (2003). New frontiers in the future of aging: From successful aging of the young old to the dilemmas of the fourth age. Gerontology, 49(2), 123-135.

Bengtson, V. L., \& Dowd, J. J. (1981). Sociological functionalism, exchange theory and life-cycle analysis: A call for more explicit theoretical bridges. The International Journal of Aging and Human Development, 12(1), 55-73. 
Bengtson, V. L., \& Roberts, R. E. (1991). Intergenerational solidarity in aging families: An example of formal theory construction. Journal of Marriage and the Family, 53(4), 856-870.

Berkman, L. F., Melchior, M., Chastang, J.-F., Niedhammer, I., Leclerc, A., \& Goldberg, M. (2004). Social integration and mortality: A prospective study of French employees of Electricity of France-Gas of France: The GAZEL Cohort. American Journal of Epidemiology, 159(2), 167-174.

Brandt, M., Deindl, C., \& Hank, K. (2012). Tracing the origins of successful aging: The role of childhood conditions and social inequality in explaining later life health. Social Science and Medicine, 74(9), 1418-1425.

Bruandet, A., Richard, F., Bombois, S., Maurage, C., Masse, I., Amouyel, P., \& Pasquier, F. (2008). Cognitive decline and survival in Alzheimer's disease according to education level. Dementia and Geriatric Cognitive Disorders, 25(1), 74-80.

Carstensen, L. L. (1993). Motivation for social contact across the life span: A theory of socioemotional selectivity. Paper presented at the Nebraska symposium on motivation.

Chi, I., \& Chou, K. (2000). Depression predicts cognitive decline in Hong Kong Chinese older adults. Aging \& Mental Health, 4(2), 148-157.

Christensen, H., Mackinnon, A., Jorm, A., Henderson, A., Scott, L., \& Korten, A. (1994). Age differences and interindividual variation in cognition in community-dwelling elderly. Psychology and Aging, 9(3), 381.

Clemens, W. (2008). Zur ,ungleichheitsempirischen Selbstvergessenheit "der deutschsprachigen Alter (n) ssoziologie. In H. Künemund \& K. R. Schroeter (Hrsg.), Soziale Ungleichheiten und kulturelle Unterschiede in Lebenslauf und Alter (S. 17-30). Wiesbaden: Springer VS.

Cohen, S. (2004). Social relationships and health. American Psychologist, 59(8), 676.

Cohen, S., Mermelstein, R., Kamarck, T., \& Hoberman, H. M. (1985). Measuring the functional components of social support social support. In I. G. Sarason \& B. Sarason (Hrsg.), Social support: Theory, research, and applications (S. 73-94). The Hague: Springer Martinus Nijhoff.

Crimmins, E. M. (2004). Trends in the health of the elderly. Annual Revue of Public Health, 25, 79-98.

Cumming, E., \& Henry, W. E. (1961). Growing old, the process of disengagement. New York: Basic Books.

den Brok, M. G., van Dalen, J. W., van Gool, W. A., Moll van Charante, E. P., de Bie, R. M., \& Richard, E. (2015). Apathy in Parkinson's disease: a systematic review and metaanalysis. Movement Disorders, 30(6), 759-769.

Domènech-Abella, J., Mundó, J., Leonardi, M., Chatterji, S., Tobiasz-Adamczyk, B., Koskinen, S., \& Haro, J. M. (2018). The association between socioeconomic status and depression among older adults in Finland, Poland and Spain: A comparative cross-sectional study of distinct measures and pathways. Journal of Affective Disorders, 241, 311-318.

Eisenmenger, M., \& Emmerling, D. (2011). Amtliche Sterbetafeln und Entwicklung der Sterblichkeit. Wirtschaft und Statistik, 3(2011), 219-238.

Ellwardt, L., van Tilburg, T., \& Aartsen, M. (2015a). The mix matters: Complex personal networks relate to higher cognitive functioning in old age. Social Science and Medicine, 125, 107-115. 
Ellwardt, L., van Tilburg, T., Aartsen, M., Wittek, R., \& Steverink, N. (2015b). Personal networks and mortality risk in older adults: A twenty-year longitudinal study. PLoS ONE, 10(3), e0116731.

Ellwardt, L., Aartsen, M., \& van Tilburg, T. (2016). Types of non-kin networks and their association with survival in late adulthood: A latent class approach. Journals of Gerontology. Series B, Psychological Sciences and Social Sciences, 72(4), 694-705.

Ferri, C. P., Prince, M., Brayne, C., Brodaty, H., Fratiglioni, L., Ganguli, M., \& Huang, Y. (2005). Global prevalence of dementia: A Delphi consensus study. The Lancet, 366(9503), 2112-2117.

Finkel, D., Reynolds, C. A., McArdle, J. J., \& Pedersen, N. L. (2007). Age changes in processing speed as a leading indicator of cognitive aging. Psychology and Aging, 22(3), 558.

Fratiglioni, L., Wang, H. X., Ericsson, K., Maytan, M., \& Winblad, B. (2000). Influence of social network on occurrence of dementia: A community-based longitudinal study. The Lancet, 355(9212), 1315-1319.

Fratiglioni, L., Paillard-Borg, S., \& Winblad, B. (2004). An active and socially integrated lifestyle in late life might protect against dementia. The Lancet Neurology, 3(6), 343-353.

Gow, A. J., Mortensen, E. L., \& Avlund, K. (2012). Activity participation and cognitive aging from age 50 to 80 in the Glostrup 1914 cohort. Journal of the American Geriatrics Society, 60(10), 1831-1838.

Han, K.-M., Han, C., Shin, C., Jee, H.-J., An, H., Yoon, H.-K., \& Kim, S.-H. (2018). Social capital, socioeconomic status, and depression in community-living elderly. Journal of Psychiatric Research, 98, 133-140.

Henretta, J. C., \& Campbell, R. T. (1976). Status attainment and status maintenance: A study of stratification in old age. American Sociological Review, 41, 981-992.

Holt-Lunstad, J., Smith, T. B., \& Layton, J. B. (2010). Social relationships and mortality risk: A meta-analytic review. PLoS Med, 7(7), e1000316.

Holt-Lunstad, J., Smith, T. B., Baker, M., Harris, T., \& Stephenson, D. (2015). Loneliness and social isolation as risk factors for mortality: A meta-analytic review. Perspectives on Psychological Science, 10(2), 227-237.

Holtzman, R. E., Rebok, G. W., Saczynski, J. S., Kouzis, A. C., Doyle, K. W., \& Eaton, W. W. (2004). Social network characteristics and cognition in middle-aged and older adults. Journals of Gerontology Series B-Psychological Sciences and Social Sciences, 59(6), 278-284.

Hoogendijk, E. O., Suanet, B., Dent, E., Deeg, D. J., \& Aartsen, M. J. (2016). Adverse effects of frailty on social functioning in older adults: Results from the Longitudinal Aging Study Amsterdam. Maturitas, 83, 45-50.

House, J. S., Kessler, R. C., \& Herzog, A. R. (1990). Age, socioeconomic status, and health. The Milbank Quarterly, 68(3), 383-411.

House, J. S., Kessler, R. C., Herzog, A. R., Mero, R. P., Kinney, A. M., \& Breslow, M. J. (1992). Social stratification, age, and health. In K. W. Schaie, D. Blazer, J. S. House, \& J. A. House (Hrsg.), Aging, health behaviors, and health outcomes (S. 1-32). Hillsdale, NJ: Erlbaum Associates.

Hughes, T. F., Andel, R., Small, B. J., Borenstein, A. R., \& Mortimer, J. A. (2008). The association between social resources and cognitive change in older adults: Evidence from the Charlotte County Healthy Aging Study. Journals of Gerontology Series B-Psychological Sciences and Social Sciences, 63(4), 241-244. 
Hultsch, D. F., Hertzog, C., Small, B. J., \& Dixon, R. A. (1999). Use it or lose it: Engaged lifestyle as a buffer of cognitive decline in aging? Psychology and Aging, 14(2), 245-263.

James, B. D., Boyle, P. A., Buchman, A. S., Barnes, L. L., \& Bennett, D. A. (2011). Life space and risk of Alzheimer disease, mild cognitive impairment, and cognitive decline in old age. The American Journal of Geriatric Psychiatry, 19(11), 961-969.

Katz, S. (1996). Disciplining old age: The formation of gerontological knowledge: Charlottesville: University of Virginia Press.

Kohli, M. (2000). Der Alters-Survey als Instrument wissenschaftlicher Beobachtung. In M. Kohli \& H. Künemund (Hrsg.), Die zweite Lebenshälfte (S. 10-32). Wiesbaden: Springer VS.

Kohli, M., Künemund, H., Motel, A., \& Szydlik, M. (2000). Soziale Ungleichheit. In M. Kohli \& H. Künemund (Hrsg.), Die zweite Lebenshälfte (S. 318-336). Wiesbaden: Springer VS.

Kuiper, J. S., Zuidersma, M., Voshaar, R. C. O., Zuidema, S. U., van den Heuvel, E. R., Stolk, R. P., \& Smidt, N. (2015). Social relationships and risk of dementia: A systematic review and meta-analysis of longitudinal cohort studies. Ageing Research Reviews, 22, 39-57.

Lampert, T., Hoebel, J., Kuntz, B., Fuchs, J., \& Nowossadeck, E. (2017). Gesundheitliche Ungleichheit im höheren Lebensalter. Paper presented at the Public Health Forum.

Lee, Y., \& Chi, I. (2016). Do cognitive leisure activities really matter in the relationship between education and cognition? Evidence from the aging, demographics, and memory study (ADAMS). Aging and Mental Health, 20(3), 252-261.

Lei, X., Sun, X., Strauss, J., Zhang, P., \& Zhao, Y. (2014). Depressive symptoms and SES among the mid-aged and elderly in China: Evidence from the China Health and Retirement Longitudinal Study national baseline. Social Science and Medicine, 120, 224-232.

Lemon, B. W., Bengtson, V. L., \& Peterson, J. A. (1972). An exploration of the activity theory of aging: Activity types and life satisfaction among in-movers to a retirement community. Journal of Gerontology, 27(4), 511-523.

Leopold, L., \& Engelhardt, H. (2011). Bildung und Gesundheitsungleichheit im Alter: Divergenz, Konvergenz oder Kontinuität? Kölner Zeitschrift für Soziologie und Sozialpsychologie, 63(2), 207-236.

Li, T., \& Zhang, Y. (2015). Social network types and the health of older adults: Exploring reciprocal associations. Social Science and Medicine, 130, 59-68.

Liberati, G., Raffone, A., \& Belardinelli, M. O. (2012). Cognitive reserve and its implications for rehabilitation and Alzheimer's disease. Cognitive Processing, 13(1), 1-12.

Litwin, H., \& Stoeckel, K. J. (2016). Social network, activity participation, and cognition: A complex relationship. Research on Aging, 38(1), 76-97.

Lleras-Muney, A. (2005). The relationship between education and adult mortality in the United States. The Review of Economic Studies, 72(1), 189-221.

Lorant, V., Deliège, D., Eaton, W., Robert, A., Philippot, P., \& Ansseau, M. (2003). Socioeconomic inequalities in depression: A meta-analysis. American Journal of Epidemiology, 157(2), 98-112.

Lubben, J. E. (1988). Assessing social networks among elderly populations. Family \& Community Health: The Journal of Health Promotion \& Maintenance, 11(3), 42-52.

Luppa, M., Sikorski, C., Luck, T., Ehreke, L., Konnopka, A., Wiese, B., ... \& Riedel-Heller, S. G. (2012). Age-and gender-specific prevalence of depression in latest-life-systematic review and meta-analysis. Journal of affective disorders, 136(3), 212-221. 
Lüscher, K. (2000). Die Ambivalenz von Generationenbeziehungen - eine allgemeine heuristische Hypothese. In M. Kohli \& M. Szydlik (Hrsg.), Generationen in Familie und Gesellschaft (S. 138-161). Opladen: Leske+Buderich.

Mackenbach, J. P., Stirbu, I., Roskam, A.-J. R., Schaap, M. M., Menvielle, G., Leinsalu, M., \& Kunst, A. E. (2008). Socioeconomic inequalities in health in 22 European countries. New England Journal of Medicine, 358(23), 2468-2481.

Maddox, G. L., \& Eisdorfer, C. (1972). Zusammenhänge zwischen Aktivität und Stimmung bei älteren Menschen. Altern - Probleme und Tatsachen. Frankfurt a. M.: Akademische Verlagsgesellschaft.

Markides, K. S., \& Machalek, R. (1984). Selective survival, aging and society. Archives of Gerontology and Geriatrics, 3(3), 207-222.

Mayer, K. U., \& Wagner, M. (2010). Lebenslagen und soziale Ungleichheit im hohen Alter. In U. Lindenberger, J. Smith, \& P. B. Baltes (Hrsg.), Die Berliner Altersstudie (S. 275299). Berlin: Akademie.

McEniry, M., Samper-Ternent, R., Flórez, C. E., Pardo, R., \& Cano-Gutierrez, C. (2018). Patterns of SES health disparities among older adults in three upper middle-and two high-income countries. The Journals of Gerontology: Series B, gby050.

McMunn, A., Nazroo, J., \& Breeze, E. (2008). Inequalities in health at older ages: A longitudinal investigation of the onset of illness and survival effects in England. Age and Ageing, 38(2), 181-187.

Meng, X., \& D'Arcy, C. (2012). Education and dementia in the context of the cognitive reserve hypothesis: A systematic review with meta-analyses and qualitative analyses. PLoS ONE, 7(6), e38268.

Mourao, R. J., Mansur, G., Malloy-Diniz, L. F., Castro Costa, E., \& Diniz, B. S. (2016). Depressive symptoms increase the risk of progression to dementia in subjects with mild cognitive impairment: systematic review and meta-analysis. International journal of geriatric psychiatry, 31(8), 905-911.

Müller, B., \& Kropp, P. (2011). Aktivität und Kognition im höheren Lebensalter - Befunde aus der ILSE: oder: Wie bleibt das Gehirn möglichst lange frisch? In J. Höppner, D. Schläfke, \& J. Thome (Hrsg.), Impulse für Psychiatrie, Psychotherapie und Psychosomatik in der Lebensspanne (S. 215-228). Berlin: Medizinisch Wissenschaftliche Verlagsgesellschaft.

Müller, B., \& Kropp, P. (2012). Freizeit im höheren Lebensalter. Ein Bereich aktiver Lebensgestaltung. Befunde aus der Interdisziplinären Längsschnittstudie des Erwachsenenalters (ILSE). In M. Kumlehn \& A. Kubik (Hrsg.), Konstrukte gelingenden Alterns (S. 207-228). Stuttgart: Kohlhammer.

Müller, B., Nienaber, C. A., Reis, O., Kropp, P., \& Meyer, W. (2014). Sexuality and affection among elderly German men and women in long-term relationships: results of a prospective population-based study. PLoS ONE, 9(11), e111404.

Neugarten, B., Havighurst, R., Munnichs, J., \& Thomae, H. (1969). Adjustment to retirement. Assen, The Netherlands: Van Gorcum.

Nilsson, C. J., Avlund, K., \& Lund, R. (2010). Social inequality in onset of mobility disability among older Danes: The mediation effect of social relations. Journal of Aging and Health, 22(4), 522-541.

Olofsson, J., Padyab, M., \& Malmberg, G. (2018). Health disparities in Europe's ageing population: The role of social network. Global Health Action, 11(1), 1445498. 
Pakpahan, E., Hoffmann, R., \& Kröger, H. (2017). The long arm of childhood circumstances on health in old age: Evidence from SHARELIFE. Advances in Life Course Research, 31, 1-10.

Reischies, F. M., \& Lindenberger, U. (2010). Grenzen und Potentiale kognitiver Leistungsfähigkeit im Alter. In U. Lindenberger, J. Smith, \& P. B. Baltes (Hrsg.), Die Berliner Altersstudie (S. 375-401). Berlin: Akademie.

Rueda, S., \& Artazcoz, L. (2009). Gender inequality in health among elderly people in a combined framework of socioeconomic position, family characteristics and social support. Ageing \& Society, 29(4), 625-647.

Saczynski, J. S., Pfeifer, L. A., Masaki, K., Korf, E. S., Laurin, D., White, L., \& Launer, L. J. (2006). The effect of social engagement on incident dementia: The Honolulu-Asia Aging Study. American Journal of Epidemiology, 163(5), 433-440.

Saraceno, C. (2010). Social inequalities in facing old-age dependency: A bi-generational perspective. Journal of European Social Policy, 20(1), 32-44.

Scarmeas, N., Levy, G., Tang, M.-X., Manly, J., \& Stern, Y. (2001). Influence of leisure activity on the incidence of Alzheimer's disease. Neurology, 57(12), 2236-2242.

Scarmeas, N., \& Stern, Y. (2003). Cognitive reserve and lifestyle. Journal of Clinical and Experimental Neuropsychology, 25(5), 625-633.

Schöllgen, I., Huxhold, O., \& Tesch-Römer, C. (2010). Socioeconomic status and health in the second half of life: findings from the German ageing survey. European Journal of Ageing, 7(1), 17-28.

Schooler, C. (1984). Psychological effects of complex environments during the life span: A review and theory. Intelligence, 8(4), 259-281.

Schwarzbach, M., Luppa, M., Forstmeier, S., König, H. H., \& Riedel-Heller, S. G. (2014). Social relations and depression in late life-a systematic review. International Journal of Geriatric Psychiatry, 29(1), 1-21.

Seeman, T. E., \& Berkman, L. F. (1988). Structural characteristics of social networks and their relationship with social support in the elderly: Who provides support. Social Science and Medicine, 26(7), 737-749.

Shanas, E., Townsend, P., Wedderburn, D., Friis, H. K., Milhoj, P., \& Stehouwer, J. (1968). Old people in three industrial societies. New York: Atherton.

Shkolnikov, V. M., Scholz, R., Jdanov, D. A., Stegmann, M., \& Von Gaudecker, H.-M. (2007). Length of life and the pensions of five million retired German men. European Journal of Public Health, 18(3), 264-269.

Spuling, S. M., Wurm, S., Wolff, J. K., \& Wünsche, J. (2017). Heißt krank zu sein sich auch krank zu fühlen? Subjektive Gesundheit und ihr Zusammenhang mit anderen Gesundheitsdimensionen. In K. Mahne, J. K. Wolff, J. Simonson, C. Tesch-Römer \& Deutsches Zentrum für Altersfragen (Hrsg.), Altern im Wandel: zwei Jahrzehnte Deutscher Alterssurvey (DEAS) (S. 157-170). Wiesbaden: Springer VS.

Tani, Y., Fujiwara, T., Kondo, N., Noma, H., Sasaki, Y., \& Kondo, K. (2016). Childhood socioeconomic status and onset of depression among Japanese older adults: the JAGES prospective cohort study. The American Journal of Geriatric Psychiatry, 24(9), 717-726.

Tartler, R. (1961). Das Alter in der modernen Gesellschaft. Stuttgart: Enke.

Tesch-Römer, C. (2010). Soziale Beziehungen alter Menschen. Stuttgart: Kohlhammer. 
Tesch-Römer, C., \& Wurm, S. (2009). Theoretische Positionen zu Gesundheit und Alter. In K. Böhm, C. Tesch-Römer, \& T. Ziese (Hrsg.), Gesundheit und Krankheit im Alter (S. 7-21). Berlin: RKI.

Tews, H. P. (1993). Neue und alte Aspekte des Strukturwandels des Alters. In H. P. Tews (Hrsg.), Lebenslagen im Strukturwandel des Alters (S. 15-42). Opladen: Springer Westdeutscher Verlag.

Then, F. S., Luck, T., Luppa, M., Thinschmidt, M., Deckert, S., Nieuwenhuijsen, K., \& Riedel-Heller, S. G. (2013). Systematic review of the effect of the psychosocial working environment on cognition and dementia. Occupational and Environmental Medicine, oemed-2013-101760.

Unger, J. B., McAvay, G., Bruce, M. L., Berkman, L., \& Seeman, T. (1999). Variation in the impact of social network characteristics on physical functioning in elderly persons: MacArthur Studies of Successful Aging. The Journals of Gerontology Series B: Psychological Sciences and Social Sciences, 54(5), 245-251.

von dem Knesebeck, O. (2005). Die Bedeutung sozialer Beziehungen für den Zusammenhang zwischen sozialer Ungleichheit und Gesundheit im Alter. Sozial-und Präventivmedizin, 50(5), 311-318.

von dem Knesebeck, O., \& Schäfer, I. (2009). Gesundheitliche Ungleichheit im höheren Lebensalter. In M. Richter \& K. Hurrelmann (Hrsg.), Gesundheitliche Ungleichheit (S. 253-265). Wiesbaden: Springer VS.

von dem Knesebeck, O., \& Vonneilich, N. (2009). Gesundheitliche Ungleichheit im Alter. Zeitschrift für Gerontologie und Geriatrie, 42(6), 459-464.

von dem Knesebeck, O., Lüschen, G., Cockerham, W. C., \& Siegrist, J. (2003). Socioeconomic status and health among the aged in the United States and Germany: A comparative cross-sectional study. Social Science and Medicine, 57(9), 1643-1652.

Vonneilich, N., Jöckel, K.-H., Erbel, R., Klein, J., Dragano, N., Weyers, S., \& von dem Knesebeck, O. (2011). Does socioeconomic status affect the association of social relationships and health? A moderator analysis. International Journal for Equity in Health, 10(1), 43.

Vonneilich, N., Jöckel, K.-H., Erbel, R., Klein, J., Dragano, N., Siegrist, J., \& von Dem Knesebeck, O. (2012). The mediating effect of social relationships on the association between socioeconomic status and subjective health - Results from the Heinz Nixdorf Recall cohort study. BMC Public Health, 12(1), 285.

Wang, H.-X., Karp, A., Winblad, B., \& Fratiglioni, L. (2002). Late-life engagement in social and leisure activities is associated with a decreased risk of dementia: A longitudinal study from the Kungsholmen project. American Journal of Epidemiology, 155(12), 1081-1087.

Wang, H.-X., Jin, Y., Hendrie, H. C., Liang, C., Yang, L., Cheng, Y., \& Murrell, J. R. (2012). Late life leisure activities and risk of cognitive decline. Journals of Gerontology Series A: Biomedical Sciences and Medical Sciences, 68(2), 205-213.

Wilson, R., Evans, D., Bienias, J., De Leon, C. M., Schneider, J., \& Bennett, D. (2003). Proneness to psychological distress is associated with risk of Alzheimer's disease. Neurology, 61(11), 1479-1485.

Wrzus, C., Hänel, M., Wagner, J., \& Neyer, F. J. (2013). Social network changes and life events across the life span: a meta-analysis. Psychological Bulletin, 139(1), 53. 
Yan, T., Escarce, J. J., Liang, L. J., Longstreth, W. T., Merkin, S. S., Ovbiagele, B., \& Brown, A. F. (2013). Exploring psychosocial pathways between neighbourhood characteristics and stroke in older adults: The cardiovascular health study. Age and Ageing, 42(3), 391-397.

Yao, L., \& Robert, S. A. (2008). The contributions of race, individual socioeconomic status, and neighborhood socioeconomic context on the self-rated health trajectories and mortality of older adults. Research on Aging, 30(2), 251-273.

Youm, Y., Laumann, E. O., Ferraro, K. F., Waite, L. J., Kim, H. C., Park, Y. R., \& Lee, J. A. (2014). Social network properties and self-rated health in later life: comparisons from the Korean social life, health, and aging project and the national social life, health and aging project. BMC Geriatrics, 14, 102.

Zis, P., Daskalaki, A., Bountouni, I., Sykioti, P., Varrassi, G., \& Paladini, A. (2017). Depression and chronic pain in the elderly: links and management challenges. Clinical interventions in aging, 12, 709.

Open Access Dieses Kapitel wird unter der Creative Commons Namensnennung 4.0 International Lizenz (http://creativecommons.org/licenses/by/4.0/deed.de) veröffentlicht, welche die Nutzung, Vervielfältigung, Bearbeitung, Verbreitung und Wiedergabe in jeglichem Medium und Format erlaubt, sofern Sie den/die ursprünglichen Autor(en) und die Quelle ordnungsgemäß nennen, einen Link zur Creative Commons Lizenz beifügen und angeben, ob Änderungen vorgenommen wurden.

Die in diesem Kapitel enthaltenen Bilder und sonstiges Drittmaterial unterliegen ebenfalls der genannten Creative Commons Lizenz, sofern sich aus der Abbildungslegende nichts anderes ergibt. Sofern das betreffende Material nicht unter der genannten Creative Commons Lizenz steht und die betreffende Handlung nicht nach gesetzlichen Vorschriften erlaubt ist, ist für die oben aufgeführten Weiterverwendungen des Materials die Einwilligung des jeweiligen Rechteinhabers einzuholen.

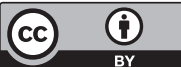

